\title{
ON THE NUMBER OF CONNECTED COMPONENTS IN THE SPACE OF CLOSED NONDEGENERATE CURVES ON $\mathbf{S}^{n}$
}

\author{
B. Z. SHAPIRO AND M. Z. SHAPIRO
}

The main definition. A parameterized curve $\gamma: \mathbf{I} \rightarrow \mathbf{R}^{n}$ is called nondegenerate if for any $t \in \mathbf{I}$ the vectors $\gamma^{\prime}(t), \ldots, \gamma^{(n)}(t)$ are linearly independent. Analogously $\gamma: \mathbf{I} \rightarrow \mathbf{S}^{n}$ is called nondegenerate if for any $t \in I$ the covariant derivatives $\gamma^{\prime}(t), \ldots, \gamma^{(n)}(t)$ span the tangent hyperplane to $\mathbf{S}^{n}$ at the point $\gamma(t)$ (compare with the notion of $n$-freedom in [G]).

Fixing an orientation in $\mathbf{R}^{n}$ or $\mathbf{S}^{n}$ we call a nondegenerate curve $\gamma$ right-oriented if the orientation on $\mathbf{S}^{n}$ induced by $\gamma^{\prime}, \ldots$, $\gamma^{(n)}$ coincides with the given one and left-oriented otherwise.

Nondegenerate curves on $\mathbf{S}^{n}$ are closely related with linear ordinary differential equations of $(n+1)$ th order. Such an equation defines two nondegenerate curves on $\mathbf{S}^{n} \subset V^{(n+1)^{*}}$, where $V^{(n+1)^{*}}$ is the $(n+1)$-dimensional vector space dual to the space of solutions as follows. For each moment $t \in \mathbf{I}$ we choose the linear hyperplane in $V^{n+1}$ of all solutions vanishing at $t$ i.e. thus obtaining a unique curve in the projective space $\mathbf{P}^{n}$ as $t$ varies. Raising it to $\mathbf{S}^{n}$ we obtain a pair of curves; both of them are right-oriented if $n$ is odd and have opposite orientations if $n$ is even (nondegeneracy follows from nonvanishing of its Wronskian).

A nondegenerate curve $\gamma:[0,1] \rightarrow \mathbf{S}^{n}$ defines a monodromy operator $M \in \mathbf{G L}_{n+1}^{+}$which maps $\gamma(0), \gamma^{\prime}(0), \ldots, \gamma^{(n)}(0)$ to $\gamma(1)$, $\gamma^{\prime}(1), \ldots, \gamma^{(n)}(1)$.

The paper [K-O] contains a complete set of invariants for symplectic leaves of the second Gelfand-Dikki bracket; namely the leaves are enumerated by pairs consisting of a monodromy operator, and a connected component of the space of right-oriented curves in the sphere with the given monodromy operator.

Received by the editors September 25, 1989 and, in revised form, November 23, 1990.

1980 Mathematics Subject Classification (1985 Revision). Primary 53A04. 
In this paper we study the number of connected components for closed nondegenerate right-oriented curves (i.e. corresponding to the identity monodromy operator). Nondegeneracy is also interesting in connection with the general theory of the $h$-principle (see $[\mathrm{G}]$ ).

Let $\mathbf{N R}^{n}\left(\mathbf{N S}^{n}\right)$ be the space of all nondegenerate closed rightoriented curves in $\mathbf{R}^{n}$ ( $\mathbf{S}^{n}$ respectively).

The question we consider is how to calculate $\pi_{0}\left(\mathbf{N S} \mathbf{S}^{n}\right)$ and $\pi_{0}\left(\mathbf{N R}^{n}\right)$. The first paper studying a similar question is [F]. Later J. Little [L1,L2] studied $\mathbf{N S}^{2}$ and $\mathbf{N R}^{3}$ and proved the following conjecture (W. Pohl): $\operatorname{card}\left(\pi_{0}\left(\mathbf{N S}^{2}\right)\right)=3$ and $\operatorname{card}\left(\pi_{0}\left(\mathbf{N R}^{3}\right)\right)=$ 2 . The invariant which distinguishes closed nondegenerate curves is an element of the fundamental group of the image of the natural map $\nu: \mathbf{N R}^{n} \rightarrow \mathbf{S O} \mathbf{O}_{n}$, where $\nu(\gamma(t))$ is the matrix obtained by orthogonalization of $\gamma^{\prime}(t), \ldots, \gamma^{(n)}(t)$; and $s \nu: \mathbf{N S}^{n} \rightarrow \mathbf{S O}_{n+1}$, where $s \nu(\gamma(t))$ is the matrix obtained by orthogonalization of $\gamma^{\prime}(t), \ldots, \gamma^{(n)}(t)$ considered as vectors in $\mathbf{R}^{n+1}$ via the standard embedding $\mathbf{S}^{n} \subset \mathbf{R}^{n+1}$.

U. Hamenstadt $[\mathrm{H}]$ continued the study of this question and formulated the following erroneous result (mentioned in [G]).

Proposition. $\left.\pi_{0}\left(\mathbf{N R}^{k}\right)=\pi_{0}(\mathbf{N S})^{k}\right)$ and consists of 2 elements for any $k>2$.

The correct statement which was recently proved by the second author is as follows:

Theorem. (1) $\pi_{0}\left(\mathbf{N R} \mathbf{R}^{k}\right)=\pi_{0}\left(\mathbf{N S}^{k}\right)$ and consists of 2 elements for any odd $k>2$, i.e. in this case the only invariant is $\pi_{1}(\nu)$.

(2) $\pi_{0}\left(\mathbf{N R}^{k}\right)=\pi_{0}\left(\mathbf{N} \mathbf{S}^{k}\right)$ and consists of 3 elements for any even $k>3$.

In this paper we will prove the following result:

Theorem 1. $\operatorname{card}\left(\pi_{0}\left(\mathbf{N S}^{2 k}\right)\right) \geq 3$.

We need several definitions.

Definiton 1. By a linear subspace of $\mathbf{S}^{n}$ we will denote any intersection of $\mathbf{S}^{n}$ with any linear subspace in $\mathbf{R}^{n+1}$ (where $\mathbf{S}^{n}$ is considered to be standardly embedded). Any $k$-tuple $\left(v_{1}, \ldots, v_{k}\right)$ of vectors tangent to $\mathbf{S}^{n}$ at some point $p$ defines a linear subspace in $\mathbf{S}^{n}$ as its intersection with the subspace in $\mathbf{R}^{n+1}$ spanned by $p, v_{1}, \ldots, v_{k}$. 
Definition 2. Consider a closed nondegenerate curve $\gamma$ on $\mathbf{S}^{n}$. The curve $\gamma$ will be called disconjugate if the sum of local multiplicities of its intersection over all intersection points of the curve with any linear hyperplane (if) is at most $n$ and conjugate otherwise. (Local multiplicity is the degree of the restriction of the divisor on the curve; in the considered case its equals the maximal dimension of the osculating subspace to the curve contained in the hyperplane.)

Remark 2 . One can easily see that the local multiplicity at a point of intersection for a nondegenerate curve is at most $n$. It equals the maximal dimension of the osculating subspace to the curve at this point lying in the hyperplane.

Definition 3. Denote by $\mathbf{S}^{n^{*}}$ the sphere dual to the considered $\mathbf{S}^{n}$, i.e. the set of all oriented hyperplanes on $\mathbf{S}^{n}$. If $\gamma$ is a nondegenerate curve on $\mathbf{S}^{n}$ then we can define the curve $\gamma^{*} \in \mathbf{S}^{n^{*}}$ dual to $\gamma$ as the set of all right-oriented osculating hyperplanes tangent to $\gamma$.

Remark 3. We are able now to give the dual formulation of disconjugacy. Namely for the curve $\gamma^{*}$ dual to the disconjugate curve $\gamma$ and an arbitrary point $x \in\left(\mathbf{S}^{n^{*}}\right)$ the sum of tangency orders over all hyperplanes passing through $x$ and tangent to $\gamma$ is at most $n$.

Notation. $\mathbf{N D}^{2 k}\left(\mathbf{N C}^{2 k}\right)$ will denote the space of all closed disconjugate (resp. conjugate) curves on $\mathbf{S}^{2 k}$.

One can see that $\mathbf{N D}^{2 k}$ is nonempty. For example, the properties of trigonometrical polynomials imply that it contains the curve whose coordinates are

$$
\sqrt{\frac{1}{k+1}}(1, \sin t, \cos t, \sin 2 t, \cos 2 t, \ldots, \sin k t, \cos k t)
$$

(this curve lies on standard $\mathbf{S}^{2 k}$ ).

Since $\mathbf{N C}^{2 k}$ consist of at least 2 components differing by the element $\pi_{1}(\nu) \in \pi_{1}\left(\mathbf{G L}_{2 k+1}\right)$ (one can easily construct two curves with trigonometrical coordinates realizing different elements of $\pi_{1}\left(\mathbf{G L}_{n}^{+}\right)$. Theorem 1 follows from the next result.

Lemma 1. $\mathbf{N D}^{2 k}$ is disconnected with $\mathbf{N C}^{2 k}$. 
The proof is divided into 2 parts:

(1) $\mathbf{N D}^{2 k}$ is open;
(2) $\mathrm{NC}^{2 k}$ is open.

Instead of (1) we will prove the following more general fact. For any $m \geq n$ the set $\mathbf{N S}_{m}^{n}$ of all nondegenerate closed curves whose sum of intersection multiplicities with any hyperplane does not exceed $m$ is open. Let $t_{1}, \ldots, t_{p}$ be pairwise different moments of intersection of $\gamma$ with an arbitrary hyperplane $\mathbf{L}$ and $1 \leq$ $k_{1}, \ldots, k_{p} \leq n$ be the set of corresponding local multiplicities $\left(\sum k_{i} \leq m\right)$. Since $\gamma$ is nondegenerate, then by the definition of multiplicity for each $t_{i}$ there exist $\delta_{i}$ and $\xi_{i}$ such that if $\mid \gamma-$ $\left.\bar{\gamma}\right|_{\mathbf{C}^{n}} \leq \xi_{i}$ and the sum $\sharp$ of intersection multiplicities of $\bar{\gamma}$ with $\mathbf{L}$ if $\bar{\gamma}$ belongs to the $\delta_{i}$-neighborhood of $\gamma\left(t_{i}\right)$ is at most $k_{i}$; (the lower index $\mathbf{C}^{n}$ means that the distance is taken with respect to the metric with $n$ derivatives). Let $U_{i}$ denote the $\delta_{i}$-neighborhoods of the points $\gamma\left(t_{i}\right)$. Denote $\bar{\xi}=\min \left\{\xi_{1}, \ldots, \xi_{p}\right\}$ and let $\rho$ be the distance between $\gamma \backslash\left\{U_{1}, \ldots, U_{p}\right\}$ and $\mathbf{L}$ in the ordinary metric of $\mathbf{R}^{n}$. Finally, take $\xi=\min \{\bar{\xi}, \rho / 2\}$; we see that if $|\gamma-\bar{\gamma}|_{\mathbf{C}^{n}}<$ $\xi / 2$ then for any hyperplane $\mathbf{L} \sharp(\mathbf{L} \cap \bar{\gamma}) \leq m$.

The idea of the proof of (2) is as follows. A hyperplane $\mathbf{L}$ is called conjugate relative to a conjugate curve $\gamma \subset \mathbf{S}^{n}$ if the sum of local intersection multiplicities of $\mathbf{L}$ with $\gamma$ exceeds $n$. If a curve is conjugate, i.e. there exists a conjugate hyperplane then by the result of Sherman [S] there exists a hyperplane with $\geq 2 k$ transversal intersections. Consequently, for any sufficiently small deformation $\bar{\gamma}$ of $\gamma$ the same hyperplane also intersects $\bar{\gamma}$ transversally at least $2 k$ times. Thus $\bar{\gamma}$ is also conjugate and $\mathrm{NC}^{2 k}$ is open.

The geometrical ideal of the proof of Sherman's result is as follows.

If $\gamma: \mathbf{I}=[a, b] \rightarrow \mathbf{S}^{n}$ is a conjugate nondegenerate curve then there exists a conjugate hyperplane $\mathbf{L}$ such that the intersection points of $\mathbf{L}$ and $\gamma$ lie on $[a, b)$. As above let $\gamma\left(t_{1}\right), \gamma\left(t_{2}\right), \ldots$, $\gamma\left(t_{p}\right)$ be the set of pairwise different intersection points of $\gamma$ and $\mathbf{L}$ and let $k_{1}, k_{2}, \ldots, k_{p}$ be the set of multiplicities of intersections. For any intersection point $\gamma\left(t_{i}\right), t_{i} \in[a, b)$ with multiplicity of intersection $k_{i}>1$ we can consider the $\left(k_{i}-1\right)$ dimensional linear system of hyperplanes preserving intersection points $\gamma\left(t_{1}\right), \gamma\left(t_{i-1}\right), \gamma\left(t_{i+1}\right), \ldots, \gamma\left(t_{p}\right)$ but with different multi- 
plicities $\tilde{k}_{1}, \tilde{k}_{i-1}, \tilde{k}_{i+1}, \ldots, \tilde{k}_{p}$ where $\sum_{j} \tilde{k}_{j}=n-k_{i}$. Then in this family of hyperplanes we can find a hyperplane $\tilde{\mathbf{L}}$ having the same multiplicities of intersections $\tilde{k}_{1}, \tilde{k}_{i-1}, \tilde{k}_{i+1} \ldots, k_{p}$ where $\sum_{j} \tilde{k}_{j}=n-k_{i}$ at the points $\gamma\left(t_{1}\right), \gamma\left(t_{i-1}, \gamma\left(t_{i+1}\right), \ldots, \gamma\left(t_{p}\right)\right.$ and with $k_{i}$ simple zeros in the neighborhood of $\gamma\left(t_{i}\right)$.

Conjecture. The space NS $^{n}$

(1) for even $n$ is homotopically equivalent to the space of all closed curves on $\mathbf{S O}_{n}$ passing through the base point;

(2) for odd $n$ consists of two parts one of which is contractible and consists of (nonstrictly) disconjugate curves and the other of which is homotopically equivalent to the space of all closed curves on $\mathbf{S O}_{n}$ passing through the base point.

\section{REFERENCES}

[G] M. L. Gromov, Partial differential relations, Springer-Verlag, Berlin and New York, 1986.

[L1] J. Little, Nondegenerated homotopies of curves on the unit 2-sphere, J. Differential Geom. 4 (1970), 339-348.

[L2] _ Third order nondegenerate homotopies of the space curves, J. Differential Geom. 5 (1971), 503-515.

[H] U. Hamenstadt, Zur Theorie von Carno-Caratheodory Metriken und ihren Anwedungen, Bonner Math. Schr. 180 (1987), 1-64.

[S] T. L. Sherman, Conjugate points and simple zeros for ordinary linear differential equations, Trans. Amer. Math. Soc. 146 (1969), 397-411.

[F] W. Fenchel. Über Krummung und Windung geschlossener Raumkurven, Math. Ann. 101 (1929), 238-252.

[K-O] B. A. Khesin and V. Yu. Ovsienko, Symplectic leaves of Gelfand-Dikii's brackets and homotopical classes of nondegenerate curves, Funkcional. Anal. i Priložen. 24 (1990), 38-47.

Department of Mathematics, University of Wisconsin, Madison, WISCONSIN 53706

UL. Molostowylch, D. 15, KoR. 5, KV. 135, Moscow, USSR, 111558 
